\title{
NOTAS
}

\section{LOS PREFACIOS A LAS EPISTOLAS DE SAN PABLO EN EL MS. ESCURIALENSE 1.1.2}

Una interesante característica de las Biblias medievales españolas es la presencia en ellas de una serie de elementos extra-bíblicos: prólogos, genealogías, epactas, cánones, notas, cartas, orden de los libros, comparaciones paralelas del contenido, sumarios y cronologías. Este hábito, sin embargo, no fue iniciado por los traductores españoles: los textos latinos de donde proceden las versiones contienen ya un buen número de piezas preliminares. Las compilaciones más completas de estos materiales latinos se deben a Donatien De Bruyne (Préfaces de la Bible latine, Namur, 1920; Sommaires, divisions et rubriques de la Bible latine, Namur, 1914) ${ }^{2}$. El ms. escurialense 1.1.2, de finales del siglo xıI o comienzos del $\mathrm{XIV}^{2}$, nos ofrece un buen ejemplo de cómo se mantuvo dicha tradición en España. Hay en este manuscrito una Biblia romanceada que constituye las Partes IV y V de la General estoria alfonsí. Nueve piezas preliminares preceden aquí a la Epístola a los Romanos: una invocación, una lista de las epistolas, otra de santos mencionados, otra que enumera 48 prucbas y testimonios, un resumen de los capitulos, un prólogo, un argumento y dos advertencias más. Las piezas preliminares abarcan algo más de 9 columnas de unas 61 líneas cada una (fols. 21lr-

1. Véase también SAMuel Berger, Les préfaces jointes aux liures de la Bible dans les manuscrits de la Vulgate, Paris, 1902; FrIdericus StegmüLLER, Repertorium biblicum Medii Aevi, t. 1: Initia biblica, Apocrypha, Prologi, Madrid, 1950; La Vetus Latina Hispana, ed. Teófilo Ayuso Marazuela, t. 1: Prolegómenos, Madrid, 1953; y T. Ayuso Marazuela, "Los elementos extra-bíblicos de la Vulgata", $E B, 2$ (1943), 139-187 [introducción]; 4 (1945), 35-60 [Octateuco], 259-269 [Reyes]; 5 (1946), 5-40 [Paralipómenos, Esdras, Tobías, Judit y Ester], 429-458 [Job y el Salterio]; 6 (1947), 187-223 [Sapienciales], 347-402 [Profetas]; 7 (1948), 147-166 [Macabeos y apéndices del Antiguo Testamento].

2 Según José Llamas, Biblias medievales romanceadas. Biblia medieval romanceada judio-cristiana, t. 1: Génesis-Reyes, Madrid, 1950, p. L, el ms. 1.1 .2 es de fines del siglo xın; según S. Berger, "Les Bibles castillanes", Ro, 28 (1899), p. 567, es del xıv; según Rafael Lapesa (conversación personal, Madison, Wisc., 28 de mayo de 1956), es de fines del xir o comienzos del xiv, fecha que coincide con la propuesta por A. G. SolAlinde, introd. a la Primera parte de la General estoria, Madrid, 1930, p. xix. Según Margherita Morreale, "La Epístola de San Pablo a los Romanos", $R A B M, 63$ (1957), 424-425, la traducción es de la época alfonsina y se acabó yrobablemente antes del año 1270. 
213v). Como esta porción del texto no ha llegado a publicarse, hemos creído oportuno hacerlo en las páginas que siguen ${ }^{3}$.

Conviene señalar varios hechos en torno a las piezas mencionadas. En primer lugar, la letra gótica en que están escritas es idéntica a la de la Epístola a los Romanos, y todo parece obra de la misma mano. La lengua parece ser también de la misma época que la de la Epístola: en ésta y en los prólogos hay casos análogos de inversión en el orden de las palabras, de apócope, de imperfectos en -ie y en -ia, de pluscuamperfectos de indicativo en $-r a$, de condicionales en -ie y de adverbios en -mjent y -mjentret.

En segundo lugar está el problema de atribución. La $6^{\mathrm{a}}$ y la $7^{\mathrm{a}}$ de las piezas prologales del ms. escurialense se atribuyen a San Jerónimo. La $6^{\text {a }}$ se intitula "El primer prologo de Sant Jheronjmo en la epistola de los romanos" y la $7^{\mathrm{a}}$, "El argumento de Sant Jheronimo sobre la discordia que aujen los del linage de los judios con los del linage de los gentiles". Ambas, sin embargo, se deben a la pluma de Pelagio, teólogo britano de finales del siglo iv y comienzos del v que publicó un texto y comentario de la Vulgata antes del año 4105. La última pieza, que contiene nuevas aclaraciones sobre las razones que tuvo el Apóstol para escribir a los romanos, carece de título y de indicación de autor en la versión española; pero según DE BRUYNe (Préfaces, p. 235) y Wordsworth-WHite (Nouum Testamentum, Pars secunda, Epistolae paulinae, Oxford, 1941, pp. 41-42), el texto latino de donde se tradujo es uno de los prólogos marcionitas, y se encuentra en la mayoría de los textos autorizados de la Vulgata ${ }^{6}$.

3 Se han llevado a cabo las siguientes transcripciones: de la Epístola a los Romanos por M. Morreale, RABM, 63 (1957) , 423-452, y por ANita Dolores Brown, Linguistic analysis of St. Paul's Epistle to the Romans and prologues to the Epistles in Ms. I.l.2 of the Escorial, tesis doctoral inédita presentada en la Universidad de Wisconsin, 1956, pp. 1-20; de la I a los Corintios por M. Morreale y C. W. Gardine, $A S T, 29$ (1957) , 273-311; y del Evangelio de San Juan, 4:1-29 por Joś́ Llamas, CD, 162 (1950), 128-130. Existe una utilísima guía elaborada por M. Morreale, "Apuntes bibliográficos para la iniciación al estudio de las traducciones bíblicas medievales en castellano", Sef, 20 (1960), 66-109.

4 Algunos ejemplos: me non demandauan (preliminares) $=$ me non preguntauan (Rom., 10:20); fuel contado (prels. y Rom., 4:3); auje (prels. y Rom., 4:19); el auia (prels.) = yo viuja (Rom., 7:9); viera (prels.) = prometiera (Rom., 1:2); siruirie (prels. y Rom., 9:12); manifiesta mjentre (prels. y Rom., 2:28); corporal mjent (prels.) = engannosa mjent (Rom., 3:13).

5 Véase Alexander Souter, "The commentary of Pelagius on the Epistles of Paul: the problem of its restoration", Proc. of the British Acad., 1905-06, p. 410 El prólogo, con una omisión importante ("Epistulam sane quae... sunt scriptae sermone") y algunas diferencias ortográficas insignificantes, se incluye entre los escritos de Rabano Mauro ( $P L$, t. 11I, cols. 1275-1278). El problema de la transmisión de este prólogo _-_quizá a través de Rabano Mauro, que fue discípulo de Alcuino, o quizá por otros medios - es interesante, pero no podemos estudiarlo aquí.

6 Marción, nacido probablemente en el Ponto, se convirtió al cristianismo y llegó a Roma hacia el año 140. Pocos años después fundó su propia iglesia, que tenía como rasgo distintivo la concepción dualista de Dios: el Dios justo y el Dios bueno. Se le considera como el primero que emprendió un estudio crítico del canon y texto del Nuevo Testamento. (The Oxford dict. of the Christian Church, ed. F. L. Cross, London, 1957, p. 854). 
En tercer lugar, la palabra "Cómpoto" es característica del texto español; aparece a continuación de cada uno de los "testimonios", desde el núm. 2 hasta el 48. En el núm. I se lee: “... que fallaredes al cuento de..."; el resto de la línea ha quedado en blanco, con espacio para unas 20 letras. No se dice, pues, de qué "cuento" o "cómputo" se han tomado los testimonios. Antonio G. Solalinde, tras de referirse a los muchos "cómputos" cronológicos que se editaron en la Edad Media, concluye que es imposible determinar con precisión cuál se utilizó en la compilación alfonsina ${ }^{7}$. Pero cabe preguntarse si la palabra compoto se refiere aquí a una tabla cronológica, ya que en una lista de testimonios escriturales el factor tiempo no viene al caso.

En cuarto lugar, los modelos de seis de las ocho piezas preliminares se pueden encontrar en tres mss. latinos de la familia ibérica de textos de la Vulgata anteriores a la versión del ms. I.1.2: el Codex Cavensis, el Codex Burgensis y el Codex Toletanus o Biblia Hispalense ${ }^{8}$. Los hemos estudiado, a través de reproducciones fotostáticas, y hemos encontrado que los tres coinciden con el ms. del Escorial en la lista de santos, en la de pruebas y testimonios, en el prólogo y el argumento del pseudo-Jerónimo y en las dos series de razones que tuvo San Pablo para escribir su epístola a los romanos. Transcribimos en el apéndice cuatro de estas seis piezas latinas y damos las variantes más significativas. Los mss. latinos incluyen asimismo unos resúmenes que tienen cierto parecido con el del ms. 1.1.2, pero su relación con el texto castellano no es muy clara, y así los excluimos de nuestro estudio. El Codex Toletanus es, de los tres mss. latinos ibéricos, el único que trae una lista de las epístolas de San Pablo, pero, a diferencia del texto romanceado, es una simple enumeración. Ninguno de los mss. latinos trae la invocación inicial del ms. castellano: "En el nombre de Dios. Amen" (fol. 211a).

Los mss. latinos, por su parte, tienen otros prólogos que no se en-

7 Antonio G. Solalinde, "Fuentes de la General estoria de Alfonso el Sabio", $R F E, 23$ (1936), p. 121.

8 El Codex Cavensis se escribió en el siglo $\mathrm{Ix}$, "probably in the middle or even past it", según E. A. Lowe, "The Codex Cavensis. New light on its later history", Quantulacumque, London, 1937, p. 328; "de fines del siglo virr, o lo más tarde, de principios del siglo Ix", según T. Ayuso Marazuela, "La Biblia visigótica de la Cava dei Tirreni”, $E B, 15$ (1956), p. 57. Los preliminares se encuentran en los fols. 253r-255v. El ms. se conserva en la abadía de la Santissima Trinità, Cava dei Tirreni (Salerno). - El Codex Burgensis es de la primera mitad del siglo $x$, según A. ANDrés, "La Biblia visigoda de San Pedro de Carderia”", $B A H, 60$ (1912), p. 107. Los prefacios están en los fols. $369 \mathrm{v}-372 \mathrm{v}$. E1 ms. se guarda en el Museo Catedralicio de Burgos. - El Codex Toletanus o Biblia Hispalense se escribió en el siglo $x$, antes del año 988, según Martín de la TORre y Pedro Longas, Catálogo de los códices latinos, t. 1: Bíblicos, Madrid, 1935, p. 1; según Agustín Millares Carlo, "De paleografía visigótica: a propósito del Codex Toletanus", RFE, 12 (1925), 252-270, es del siglo $x$ y una parte de él se escribió en la primera mitad de ese siglo. La porción que nos interesa está en los fols. $321 \mathrm{r}-325 \mathrm{v}$. E1 ms. se conserva en la B.N.M. -Teníamos asimismo la intención de analizar el Codex Complutensis, pero según una carta de María Teresa de la Peña (Archivo Histórico Nacional, Madrid, 15 de noviembre de 1961), el ms. no estaba en condiciones de ser fotografiado. 
cuentran en el escurialense, a saber: $A$ ) "Prologum subter adjectum... perlegerit"; $B$ ) "Multis oecupatus necessitatibus... corrigerentur mentes", que sigue inmediatamente al anterior; $C$ ) "Deus uerax est... subiectum non erit", los cánones llamados de Primus. El Toletanus incluye otras dos piezas: D) "Iam dudum saulus procerum...", que el ms. intitula "uersi pape damasi episcopi", y E) "O mirum textus... numeri septenariam". La razón por la cual estos últimos elementos faltan en la versión española sería materia de especulación y de mayor estudio. Todas las piezas prologales, como observa De Bruyne, sirven de indicios para colegir la fecha y el lugar en que se escribieron las diversas recensiones de textos de la Vulgata a lo largo de la Edad Media $^{9}$.

El siguiente cuadro muestra el orden de los preliminares en los distintos mss.:

\section{Ms. I.l.2}

I. Invocación

2. Lista de epístolas

3. Lista de santos

4. Las 48 pruebas

5. Resumen de los capítulos

6. Primer prólogo

7. Argumento

8. Razones del envío

9. Más razones

\section{Cavensis Burgensis Toletanus}

$\begin{array}{lll}\text { A } & 6 & \text { A } \\ \text { B } & \text { A } & \text { B } \\ \text { C } & \text { B } & \text { D } \\ 5(?) & \text { C } & 2(?) \\ 4 & 7 & \text { E } \\ 3 & 9 & \text { C } \\ 6 & 8 & 3 \\ 7 & 3 & 4 \\ 9 & 4 & 5(?) \\ 8 & 5(?) & 6 \\ & & 7 \\ & & 9 \\ & & 8\end{array}$

(Salta a la vista la semejanza que hay entre el Toletanus y el ms. I.1.2 en cuanto al orden de los preliminares, con excepción de las dos últimas piezas).

En conclusión: las diferencias entre el ms. escurialense I.1.2 y los tres mss. latinos estudiados nos hacen pensar que ninguno de estos tres pudo haber sido el modelo único utilizado por el romanceador (o romanceadores). Pero cabe deducir, a título de hipótesis, que el texto latino que sirvió de base a los prólogos de las Epístolas en el ms. escurialense tenía las siguientes características: 1) la omisión de la frase herodionem, quem cognatum uocat, en la lista de santos; 2) la adición de una forma latina del nombre Hermen después de Patroban, en la misma lista; 3) la omisión de XI. In esaia: numquid dicit figmentum ei qui se finxit, en la enumeración de pruebas; 4) la adición, en la misma

9 D. De Bruyne, "Etude sur les origines de la Vulgate en Espagne", $R B, 31$ (1914-19), p. 373: "L'histoire des préfaces doit être une lumière pour l'étude des textes, souvent elle ne peut indiquer ni les sources utilisées par un recenseur, ni les principes qui l'ont guidé, mais elle peut dater et localiser des recensions, qui par l'examen des seules variantes restent plus ou moins imprécises". 
serie de pruebas, de las palabras latinas correspondientes a "La xviij" es en este xjo capitulo: Los que fincaren dellos seran saluos. Ysaya", a continuación de si fuerit numerus filiorum israhel; 5) la presencia, en el primer prólogo, de un texto latino de donde proceda la frase "Cjerta $e t$ manifiesta cosa es que por tirar las dubdas en que los omnes son et por seer ende mas ciertos de las cosas en que dubdan, son fechas las exposiciones $e t$ los declaramjentos. Por ende deuemos entender... esta demanda"; 6) la colocación de colosenses antes de thessalonicenses, en el mismo prólogo; 7) la adición, en el argumento, de una frase latina correspondiente a "catastes los ydolos", después de "soliedes ueer a Dios"; 8) la adición de una frase latina correspondiente a "mudaron la su gloria en figuras fechas por mano", en el mismo argumento; 9) el nombre athenis en lugar de corintho, en la última línea.

University of Arizona.

Dolores Brown

\section{APÉNDICE A}

\section{[Invocación]}

[211a] En el nombre de Dios. Amen.

\section{[Lista de epistolas]}

Aqui comjença el ordenamjento de las xiiij epistolas de Sant Paulo et a quales gentes las embio.

Primera mjentre embio el epistola, la primera destas xiiij epistolas, a los romanos. Son en Ythalia. La $1^{\text {a }}$ ij.

Et a los chorinthios. Son de Achaya.

Et a los de Galathas o Galacia. Son de Grecia. I.

Et a los de Epheso. Son en Asya. 1. Et a $l^{*}$ philippenses o philipianos, en Macedonia. $1^{\text {a }}$

Et a los colosenses que son en Asya, en Laodicia. 1:

Et a los de Thesalonica, en Macedonja. ij.

Et a Timotheo, ij.

Et a Thitho. 1.

Et a Philemon. 1.

Et a los hebreos. 1.

[Lista de santos]

Otrossi estos son los sanctos que

* los] + de nombra Sant Paulo en la su epistola primera que embia a los romanos, en la fin* delia: luego al comjenço em. bia a saludar a los de donna Phebo assi como hermana. Desi llama et nombra a las hermanas** et embia las el saludar et encomendar a Priscilla o Prisca et a Aguila. Et gradesce les mucho lo que ellas fizieron et siruieron por amor de Dios. Et esso mismo a Pheneto, su amigo, el primero creyente en Christo que fue en Asia, et a Maria que trabaio mucho por Dios, et a Andronico et a Junia, sus cormanos. Et llama los catiuos et nobles en la predicacion et que fueran ante que el en la fe de Christo. Et otrossi embia saludar a Ampliaco, su amigo, et a Vrban, su ayudador, et a Stath, su amigo, et Apelles, el bueno por Ghristo, et a Triphena et a Triphora et a Perssida, que trabaiaron*** mucho por Dios. Et otrossi embia saludar a Rupho, escogido por Dios, et a su madre et suya, et a Ansiecreto, et a Phlegothan, et a Hermen, et a Patroban, et a Herman, et a Philiogo, et a Julian, et a Nereo et

* fin + de

*: hermanas] hermana

***: trabaiaron] trabaron 